\title{
Bifidobacterium animalis subsp. lactis BB-12 in reducing the risk of infections in infancy
}

\author{
Teemu Taipale ${ }^{1 *}$, Kaisu Pienihäkkinen ${ }^{2}$, Erika Isolauri ${ }^{3}$, Charlotte Larsen ${ }^{4}$, Elke Brockmann ${ }^{4}$, \\ Pentti Alanen ${ }^{2}$, Jorma Jokela ${ }^{1}$ and Eva Söderling ${ }^{2}$ \\ ${ }^{1}$ Korpilahti-Muurame Health Care Center, 40950 Muurame, Finland \\ ${ }^{2}$ Institute of Dentistry, University of Turku, 20520 Turku, Finland \\ ${ }^{3}$ Department of Paediatrics, University of Turku, 20014 Turku, Finland \\ ${ }^{4}$ Chr. Hansen A/S, 2970 Hoersholm, Denmark
}

(Received 2 March 2010 - Revised 20 July 2010 - Accepted 17 August 2010 - First published online 24 September 2010)

\section{Abstract}

The impact of controlled administration of Bifidobacterium animalis subsp. lactis BB-12 (BB-12) on the risk of acute infectious diseases was studied in healthy newborn infants. In this double-blind, placebo-controlled study, 109 newborn 1-month-old infants were assigned randomly to a probiotic group receiving a BB-12-containing tablet $(n 55)$ or to a control group receiving a control tablet $(n 54)$. Test tablets were administered to the infants twice a day (daily dose of BB-12 10 billion colony-forming units) from the age of $1-2$ months to 8 months with a novel slow-release pacifier or a spoon. Breastfeeding habits, pacifier use, dietary habits, medications and all signs and symptoms of acute infections were registered. At the age of 8 months, faecal samples were collected for BB-12 determination (quantitative PCR method). The baseline characteristics of the two groups were similar, as was the duration of exclusive breastfeeding. BB-12 was recovered (detection limit $\log 5$ ) in the faeces of $62 \%$ of the infants receiving the BB-12 tablet. The daily duration of pacifier sucking was not associated with the occurrence of acute otitis media. No significant differences between the groups were observed in reported gastrointestinal symptoms, otitis media or use of antibiotics. However, the infants receiving BB-12 were reported to have experienced fewer respiratory infections $(65 v$. $94 \%$; risk ratio $0.69 ; 95 \%$ CI $0.53,0.89 ; P=0.014)$ than the control infants. Controlled administration of BB- 12 in early childhood may reduce respiratory infections.

\section{Key words: Probiotics: Infants: Pacifiers: Infections: Bifidobacterium}

Infectious diseases are the most significant illnesses for infants worldwide. In developed countries, most children experience several respiratory tract infections during the first year of their life ${ }^{(1)}$. Respiratory tract infections often coincide with or precede acute otitis media (AOM), the most common cause for antibiotic use in children $^{(2)}$. Gastrointestinal (GI) infections are the second-most common diseases in childhood and even cause increased mortality in developing countries. Infections early in life assist in shaping immunological development, and consequently may both protect children and predispose them to repeated infections, inflammatory diseases and allergic diseases later in life $\mathrm{e}^{(3,4)}$.

Indigenous intestinal microbiota, the primary source of microbial exposure, are considered instrumental in providing maturational stimuli to the immature immune system $^{(5)}$. This creates the basis of the concept for probiotics, defined as live micro-organisms which when administered in adequate amounts confer a health benefit to the host ${ }^{(6)}$. Probiotics have several beneficial effects, including the normalisation of the gut microbiota composition and interaction with the innate and adaptive immune system of the child, which may promote resistance against pathogens ${ }^{(7,8)}$. Particularly, the probiotic strains Lactobacillus rhamnosus GG and Bifidobacterium lactis are recommended in treatment and prevention of childhood diarrhoeal and allergic diseases ${ }^{(9)}$. B. animalis subsp. lactis BB-12 (BB-12) was shown to enhance mucosal resistance to infections by increasing the active stimulation of local IgA production in healthy infants ${ }^{(10)}$. Previous studies have demonstrated that probiotic therapy in infancy can prevent or treat viral GI infections even better than bacterial infections ${ }^{(11)}$. In many cases, respiratory infections are viral in origin, and the respiratory tract may be an appropriate area for probiotic immunostimulation ${ }^{(8)}$.

Abbreviations: AOM, acute otitis media; BB-12, Bifidobacterium animalis subsp. lactis BB-12; GI, gastrointestinal.

*Corresponding author: Dr T. Taipale, fax +358 14266 2770, email teemu.taipale@muurame.fi 
There are several sources of confounders in probiotic interventions in children. First, delivering probiotics in a controlled way to young children can be problematic. Second, the duration of breastfeeding and the use of infant formulas affect the outcome. Our aim was to deliver to infants a probiotic tablet containing BB-12 using either a novel slow-release pacifier or a spoon and to investigate BB-12 passage through the GI tract. We have shown earlier that the probiotic tablet dissolves in a controlled way from the pouch of the pacifier, and that the administration method is feasible ${ }^{(12)}$. We hypothesised that the administration of BB-12 to young healthy children could reduce the risk of acute infectious diseases, including AOM and respiratory infections.

\section{Methods}

\section{Participants}

The families participating in this randomised, double-blind, placebo-controlled clinical trial (NCT00638677, http:// www.clinicaltrials.gov) were recruited in Muurame and Korpilahti, Finland, between September 2004 and February 2007. Pregnant mothers ( $n$ 479) received a leaflet regarding the trial at well-baby clinics. The families interested in participating ( $n$ 189) then contacted the research nurse. After receiving detailed information, 175 families gave their preliminary consent to participate in the study. After delivery, 163 families confirmed their willingness to participate. Reasons for not participating in the trial included moving out of the area, miscarriage and lack of interest in the study. The 163 newborn 1-month-old infants were assigned to three study groups by one of the authors (T. T.; Fig. 1) according to a randomisation list, which

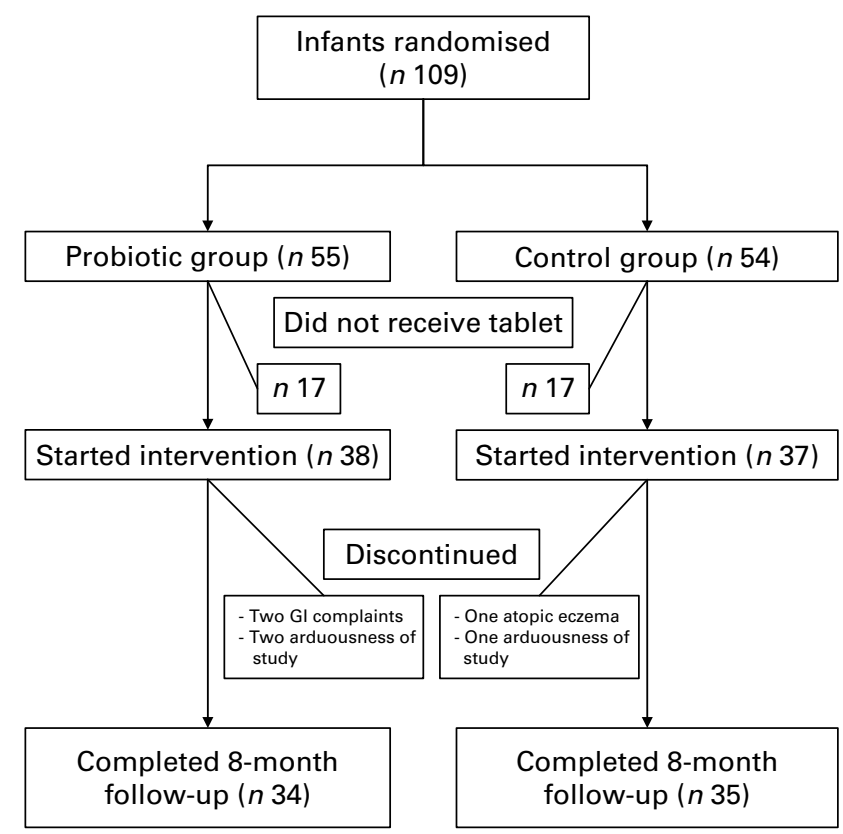

Fig. 1. Clinical trial profile. Gl, gastrointestinal. had been previously computer generated in blocks of three. The present two-armed trial is a substudy of a three-armed trial originally aiming to study the effect of BB-12 and xylitol on oral health. After the randomisation, altogether 109 children belonged to either the probiotic group (BB-12 group; $n$ 55) or the control group (xylitol control group; $n$ 54). At the 8 -month visit, the probiotic group consisted of thirty-four children, and the control group consisted of thirty-five children.

The inclusion criteria of the study were (1) the child was healthy, (2) the parents were willing to use the novel slowrelease pacifier and (3) the child started to receive the tablet before the age of 2 months. In cases where the child did not start using the pacifier but the parents were motivated to remain in the study, they were offered the possibility of delivering the crushed tablet to the child using a spoon. The present study was conducted according to the guidelines laid down in the Declaration of Helsinki, and all procedures involving human patients were approved by the Hospital District of Southwest Finland. A written informed consent was obtained from all the subjects. The ethics committee defined the stopping rules.

\section{Interventions}

The probiotic bacterium used was BB-12 (DSM 15 954; Chr. Hansen A/S, Hoersholm, Denmark). BB-12 is a safe and well-tolerated probiotic ${ }^{(13)}$. The parents were advised to give their children two probiotic tablets per day via a novel slow-release pacifier (Alanen and Söderling, US patent $6.203 .566,2000$ ). The pacifiers (Fig. 2) were manufactured by Mekalasi Oy (Konnevesi, Finland). The pacifier contains a pouch in which the tablet is inserted. Each tablet contained 5 billion colony-forming units of BB-12. Until 6-8 months of age, the children received the tablet via a small pacifier, thereafter via a larger pacifier. The tablet in the small pacifier contained $100 \mathrm{mg}$ xylitol (Danisco Limited, Kotka, Finland), the tablet in the larger pacifier contained $300 \mathrm{mg}$ xylitol, both in addition to BB-12. The control tablets contained xylitol alone. All the tablets were manufactured by Oy Karl Fazer Ab (Vantaa, Finland)

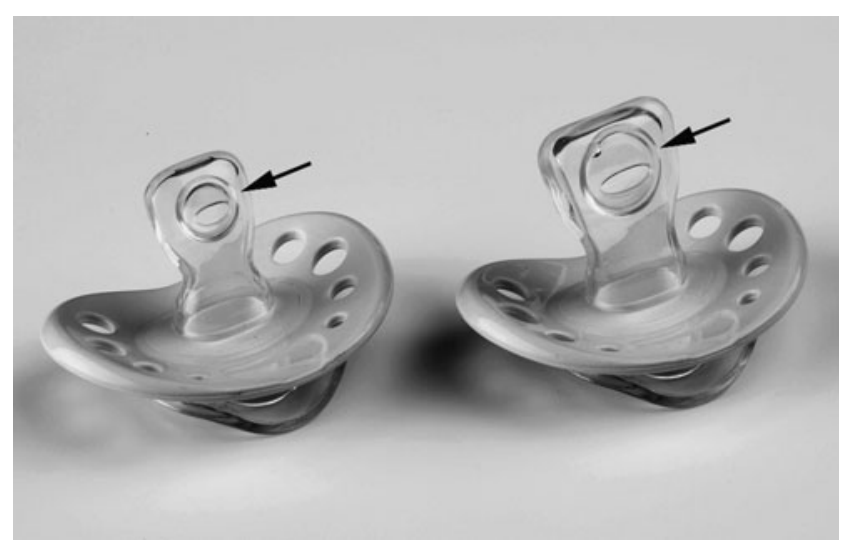

Fig. 2. The novel slow-release pacifier with pouch $(\uparrow)$. 
and packed in white plastic bottles with colour codes. Freshly prepared tablets were delivered to the families biannually and stored in a refrigerator. The families were encouraged not to use any other pacifier besides the slow-release pacifier twice a day. If, however, they wanted to use a regular pacifier as well, they were offered the pouch-free version of the slow-release pacifier (Alanen and Varrela, US patent 5.922.010, 1999).

\section{Data collection}

The infants visited the health care centres at 1 and 8 months of age. At the 1-month visit, a trained professional, a dental nurse or a dental hygienist interviewed the guardian for information on the birth and the family, as well as on breastfeeding and possible complementary feeding. In addition to reporting infectious and non-infectious diseases in the infants, the guardians also reported diseases in parents and siblings: asthma, allergies, atopic and other skin disorders, diabetes, rheumatoid diseases and GI disorders. Parents' smoking habits and special diets (lactose free, vegetarian, etc.) were also recorded. The parents were not, however, interviewed regarding the possible use of probiotic products, which in Finland have been in common use since early 1990s and the product assortment is the subject of constant change. At the time of the study, the availability of BB-12-containing products was limited, and BB-12 was found only in a few products containing undefined mixtures of bifidobacteria. At the 1-month visit, the guardian also received hands-on instructions on the use of the slow-release pacifier and study diaries.

At the 8-month examination, the parents were interviewed by a trained professional for information on breastfeeding status, use of the tablet/pacifier, use of complementary foods in the infant's diet, dairy products and products containing probiotics. The complementary foods monitored included berries, fruits, vegetables, meat, fish and grain products. All the interviews were performed using structured questionnaires validated in earlier studies $^{(3)}$. Information on infections and other health problems was recorded by the parents in special diaries ${ }^{(3)}$. The symptoms of respiratory infections included runny nose, nasal congestion, cough and shortness of breath. Respiratory infection was diagnosed when the child had at least two infectious symptoms during one day or one symptom during two consecutive days ${ }^{(14)}$. Parents also reported respiratory infections with complications (bronchitis, pneumonia and sinusitis) diagnosed by a doctor (municipal or private) unrelated to the study. The AOM occurrences reported by parents included all doctordiagnosed acute ear infections. GI infections included every episode with watery diarrhoea or vomiting. Fever episodes included elevated body temperatures $\left(>37^{\circ} \mathrm{C}\right)$ lasting for at least $1 \mathrm{~d}$. For infectious diseases and use of antibiotics and other prescribed drugs, frequency of episodes/usage was registered. Parents reported also any occurrence of atopic diseases or allergic sensitisations. In addition, symptoms of colic in the infant, i.e. crying uncontrollably for more than $3 \mathrm{~h} / \mathrm{d}$ for at least $3 \mathrm{~d}$ a week, were recorded. All adverse effects were recorded in detail. At the 8-month visit, fresh faecal samples collected by the families were delivered to the health care centres. The faecal samples were stored at the health care centres at $-20^{\circ} \mathrm{C}$ for up to 2 months and then delivered on dry ice to Turku. The faecal samples were stored in Turku at $-70^{\circ} \mathrm{C}$ before transportation on dry ice to Chr. Hansen for the analysis of BB-12.

\section{Quantification of Bifidobacterium animalis subsp. lactis} $B B-12$ in faeces by quantitative PCR

BB-12 DNA was quantified by a B. animalis subsp. lactisspecific quantitative PCR assay. BB-12 is a member of this subspecies, which is rarely encountered in human subjects who have not ingested strains of it recently. Assay design was based on partial bifidobacterial 23S ribosomal DNA sequences (GenBank accession numbers GQ340897GQ340908). Specificity of the assay was tested on DNA purified from reference ON cultures. DNA was extracted in duplicate from each faecal sample. Total DNA was extracted from approximately $200 \mathrm{mg}$ faeces with the help of the QiaAmp DNA Stool Mini Kit (Qiagen, Hilden, Germany) with the following modifications: in a screw cap tube, $200 \mathrm{mg}$ faeces were mixed with $1 \mathrm{ml}$ ASL buffer and $0.1 \mathrm{~mm}$ zirconia beads and treated $5 \mathrm{~min}$ in a MiniBeadbeater (BioSpec, Bartlesville, OK, USA) at the highest speed. Thereafter, purification was continued according to the instructions of the manufacturer. Extraction efficiency and detection limit were determined to be $5 \%$ and $10^{5}$ colony-forming units/g, respectively, using faecal samples spiked with known amounts of BB-12 colony-forming units.

For the quantitative PCR, faecal DNA was diluted tenfold. Each DNA sample was analysed in triplicate. Each PCR mixture contained $25 \mu \mathrm{l} 2 \times$ Probe Mastermix (Eurogentec, Liège, Belgium), $300 \mathrm{~nm}$ each BAL-23S-F (5'-CAGGTGGTCTGGTAGAGTATACCG- $\left.3^{\prime}\right)$ and BAL-23S-R (5'-ACGGCGACTTGCGTCTTG-3'), 250 nM BAL-23S-P (5'-FAM-CGCCCACGACCCGCAAG-TAMRA- $3^{\prime}$ ) and $5 \mu \mathrm{l}$ DNA in a total volume of $50 \mu \mathrm{l}$. Reactions were run on a ABI Prism 7500 with the following program: one cycle of $10 \mathrm{~min}$ at $95^{\circ} \mathrm{C}$ followed by $2 \mathrm{~min}$ at $50^{\circ} \mathrm{C}$, forty-five cycles of $15 \mathrm{~s}$ at $95^{\circ} \mathrm{C}$ followed by $1 \mathrm{~min}$ at $60^{\circ} \mathrm{C}$. Quantification of faecal BB-12 DNA was performed by absolute quantification against a dilution series of a pure BB-12 DNA sample.

\section{Outcome measures}

The primary outcome measures for the study were the reported cumulative incidence of acute respiratory infections and doctor-diagnosed AOM occurring before the 
age of 8 months. Successful intestinal passage of BB-12 was chosen as the secondary outcome measure.

\section{Sample size and blinding}

AOM occurs at least once in $40 \%$ of children ${ }^{(2)}$, and infants experience three to six respiratory tract infections during the first year of life ${ }^{(1,15)}$. Up to $80 \%$ of Finnish children use a pacifier. We calculated that with a group size of approximately forty children starting the intervention, we would be able to obtain not only statistically but also clinically significant results. The population in the Korpilahti and Muurame areas is rather stable, and attrition of children as a result of moving out of the area could be estimated to be low. All the study personnel and participants were blinded to treatment assignment for the duration of the study. Only one of the authors (E. S.) had the code, since she was responsible for checking the BB-12 viability of the tablet batches. She did not, however, participate in producing or analysing the data at any stage of the trial and had no contact with the study participants.

\section{Statistical methods}

Data were analysed with SPSS (version 14.0; SPSS, Inc., Chicago, IL, USA) by a blinded statistician (K. P.). In terms of baseline/background characteristics, the differences between the groups were tested for significance using the Student's $t$ test and $\chi^{2}$ test. Additionally, differences between the groups in terms of distribution in reported respiratory infection, $\mathrm{AOM}$, fever and GI infection categories were tested for significance using the $\chi^{2}$ test, for original frequency as well as for dichotomised data. The risk ratio and its $95 \% \mathrm{CI}$ were calculated to measure the group differences in relation to cumulative incidence of the studied diseases. A $P$ value of $<0.05$ was considered statistically significant (Table 1).

\section{Results}

Of the infants randomised, 69\% (75 of 109) started the novel administration method, receiving either the BB-12 ( $n$ 38) or the xylitol control ( $n$ 37). The rest of the infants, $31 \%$ (34 of 109), did not accept the pacifier before the age of 2 months, and the parents were not motivated to crush the tablet. Only those who completed the follow-up ( $n$ 69) were included in the analysis (Fig. 1). In the BB-12 group, $62 \%$ (twenty-one of thirty-four) of the infants and in the control group, 54\% (nineteen of thirty-five) of the infants received the tablet solely via the pacifier. The crushed tablet was the sole administration method for $15 \%$ (five of thirty-four) of the infants receiving the BB-12 tablet and for $11 \%$ (four of thirty-five) of the infants receiving the control tablet. The rest of the families used both the administration methods until the 8-month examination. The duration of tablet delivery did not differ between the groups $(P=0 \cdot 408)$.

Delivery of BB-12 significantly reduced the occurrence of respiratory infections reported by parents during the first 8 months of life (Table 2). Respiratory infections were frequent during the follow-up: 65\% (twenty-two of thirty-four) of the children in the BB-12 group and 94\% (thirty-three of thirty-five) in the control group had experienced one or more episodes of respiratory infections at the 8 -month examination ( $P=0.014)$. No differences between the BB-12 and control groups were detected in the occurrence of doctor-diagnosed AOM $(P=0 \cdot 455)$. The occurrence of $\mathrm{AOM}$ in the children in both the groups was low; only $26 \%$ (nine of thirty-four) in the BB-12 group and $17 \%$ (six of thirty-five) in the control group experienced any AOM episodes. No association between the occurrence of $\mathrm{AOM}$ and pacifier usage (sucking time per day, duration of usage) could be detected. Also the occurrence of GI infections was low, and no differences between the BB-12 and the control groups were detected $(P=0.605)$. No differences were found between the two

Table 1. Baseline characteristics according to the study group (Percentages, mean values and standard deviations)

\begin{tabular}{|c|c|c|c|c|c|c|c|}
\hline & \multicolumn{3}{|c|}{ Probiotic group ( $n$ 34) } & \multicolumn{3}{|c|}{ Control group ( $n$ 35) } & \multirow[b]{2}{*}{$P^{*}$} \\
\hline & $\%$ & Mean & SD & $\%$ & Mean & SD & \\
\hline \multicolumn{8}{|l|}{ Parent } \\
\hline Primapara & 53 & & & 40 & & & 0.530 \\
\hline Caesarean section & 24 & & & 35 & & & 0.338 \\
\hline Age of mothers (years) & & 31.4 & $5 \cdot 5$ & & 30.5 & $5 \cdot 3$ & 0.494 \\
\hline Age of fathers (years) & & 33.4 & $6 \cdot 1$ & & $35 \cdot 2$ & 5.7 & 0.195 \\
\hline Maternal smoking & 11 & & & 21 & & & 0.299 \\
\hline Paternal smoking & 29 & & & 26 & & & 0.728 \\
\hline \multicolumn{8}{|l|}{ Child } \\
\hline Boys & 50 & & & 57 & & & 0.552 \\
\hline Gestational age (weeks) & & $40 \cdot 0$ & $1 \cdot 1$ & & 39.7 & $1 \cdot 2$ & 0.320 \\
\hline Exclusive breastfeeding (months) & & 3.4 & 1.7 & & 3.8 & 1.9 & 0.692 \\
\hline Total breastfeeding (months) & & $7 \cdot 1$ & $2 \cdot 3$ & & $6 \cdot 9$ & $3 \cdot 1$ & 0.141 \\
\hline
\end{tabular}


Table 2. Cumulative incidence of self-reported infections and the use of antibiotics during the first 8 months of life (Percentages and number of infants; risk ratio (RR) and $95 \%$ confidence intervals)

\begin{tabular}{|c|c|c|c|c|c|c|c|}
\hline & \multicolumn{2}{|c|}{$\begin{array}{l}\text { Probiotic } \\
\text { group }(n 34)\end{array}$} & \multicolumn{2}{|c|}{$\begin{array}{l}\text { Control group } \\
\qquad(n 35)\end{array}$} & \multirow[b]{2}{*}{$\mathrm{RR}$} & \multirow[b]{2}{*}{$95 \% \mathrm{Cl}$} & \multirow[b]{2}{*}{$P$} \\
\hline & $n$ & $\%$ & $n$ & $\%$ & & & \\
\hline Respiratory infection & 22 & 65 & 33 & 94 & 0.69 & $0.53,0.89$ & 0.014 \\
\hline AOM & 9 & 26 & 6 & 17 & 1.54 & $0.62,3.87$ & 0.455 \\
\hline Fever & 16 & 47 & 14 & 40 & $1 \cdot 18$ & $0.69,2.02$ & 0.676 \\
\hline Gastrointestinal infection & 9 & 26 & 7 & 20 & 1.32 & $0.56,3.15$ & 0.605 \\
\hline Antibiotic use & 10 & 29 & 8 & 23 & 1.29 & $0.58,2.87$ & 0.535 \\
\hline
\end{tabular}

AOM, acute otitis media.

groups in the occurrence of fever episodes $(P=0.676)$ or antibiotic treatments $(P=0.535)$.

As for non-infectious diseases, parents reported colic symptoms in 6\% (two of thirty-four) of the infants in the BB-12 group and $11 \%$ (four of thirty-five) of the infants in the control group. Atopic eczema was detected in 26\% (nine of thirty-four) of the infants receiving the BB-12 tablet and 34\% (twelve of thirty-five) receiving the control tablet. Dairy milk allergy was diagnosed in three children, two in the probiotic group and one in the control group.

The groups did not differ with regard to baseline characteristics (Table 1). The age of the mothers and fathers, the educational background of the parents, the number of parents obeying special diets or occurrence of reported diseases in the families did not differ between the groups. The mean duration of exclusive breastfeeding was 3.4 (SD 1.7) months in the probiotic group and 3.8 (SD 1.9) months in the control group ( $P=0.692)$. At the age of 8 months, breastfeeding was still continuing for $44 \%$ (fifteen of thirty-four) of the infants in the probiotic group and 63\% (twenty-two of thirty-five) of the infants in the control group $(P=0 \cdot 141)$. The diet histories of the children in the two groups were similar. Five children in the control group and three children in the BB-12 group used probiotic-containing products, other than BB-12, irregularly during the intervention period.

There were no significant differences between the groups in the reported use of pacifiers. About $10 \%$ of the children changed the smaller slow-release pacifier to the larger one after the age of 6 months. Five of the children in both the groups used a commercial pacifier in addition to the novel pacifier. Two-thirds of the children in both the groups sucked on the pacifier for $1 \mathrm{~h} / \mathrm{d}$ or more, and seven children in the BB-12 group and six children in the control group did so for $3 \mathrm{~h}$ or more per day.

Faecal samples from all the children in the BB-12 and control groups were available. BB-12 was recovered in the faeces of $62 \%$ (twenty-one of thirty-four) of the infants receiving the BB-12 tablet and $17 \%$ (six of thirty-five) of the infants receiving the control tablet. All BB-12-positive children in the BB-12 group had received the tablet either via the pacifier or with a spoon until the 8-month examination. One child had received one tablet daily, all others received two tablets. Also, for the thirteen BB-12negative children in the BB-12 group, one-half of the parents reported the tablet delivery of two tablets per day until the 8-month examination.

No serious adverse effects were detected during the administration period. Two infants receiving BB-12 withdrew from the study as a result of GI complaints. One infant in the control group was diagnosed with atopic eczema, and his physician recommended the family to discontinue from the intervention.

\section{Discussion}

We found a significant reduction in the incidence of respiratory infections during the first 8 months of life by probiotic supplementation in healthy breastfed infants. With respect to the duration of exclusive and total breastfeeding, the mothers in the present study represent typical Finnish mothers. Thus, probiotics may add to the protection provided by human breast milk. Recently, the European Society for Paediatric Gastroenterology, Hepatology and Nutrition recommended exclusive breastfeeding for about 6 months to support healthy growth and development in young children ${ }^{(16)}$. In Finland, $51 \%$ of the infants, and in the study's hospital district area, $66 \%$ are exclusively breastfed at 3 months $^{(17)}$, as shown also in the present study. The duration of breastfeeding may indeed explain the low incidence of infectious diseases and also insignificant differences in infectious diseases, especially AOM, between the groups, contributing also to the low number of antibiotic prescriptions in each. Beyond providing antibodies and non-specific anti-microbial molecules, breastfeeding influences the composition of indigenous intestinal microbiota by promoting the growth of bifidobacteria ${ }^{(18)}$.

We found a reduction in the occurrence of respiratory infections with the supplementation of a single probiotic, BB-12. Weizman et $a l .{ }^{(19)}$ compared infant formulas with BB-12 or L. reuteri (ATCC 55730) in infants aged 4-10 months. They found significantly fewer febrile episodes but no effect on respiratory illnesses compared to the control group during a 12-week follow-up. In a recent study, the combination of two probiotic strains, BB-12 and 
Lactobacillus GG, reduced the risk of early AOM and recurrent respiratory infections in children requiring formula before the age of 2 months ${ }^{(15)}$. The intervention started before the age of 2 months, and the follow-up period was 12 months, longer than that in Weizman's study. Recently, also the supplementation of L. acidophilus NCFM and B. animalis subsp. lactis Bi-07 was shown to reduce the occurrence of influenza-like symptoms in healthy children ${ }^{(20)}$. The seemingly contradictory results may be explained by the fact that most of the infants in the present study received 10 billion colony-forming units BB-12 via controlled administration, but also strainspecific differences in probiotic effects and the use of combinations of probiotics may influence the results. It is obvious that the starting age and duration of intervention may be crucial in the possible beneficial effects of probiotic supplementation in early childhood. Likewise, the diet of the infant and the food matrix contribute to the outcome ${ }^{(21)}$. Hence, extrapolation of the present results to other infant populations may require further clinical demonstrations.

Up to $80 \%$ of Finnish children use a pacifier. Pacifier use has been thought to shorten the duration of exclusive breastfeeding and also to increase risk of morbidity, especially $\mathrm{AOM}^{(22-24)}$. These notions could not be confirmed here. Xylitol was chosen in the food supplement tablet to act as an optimal and tooth-friendly bulk agent. The daily dose required to prevent AOM is suggested to be 40-50 times higher than that used in the present study $^{(25)}$. Consequently, we did not expect to observe any effects of xylitol on the occurrence of AOM. The GI complaints, reported as reasons of withdrawal of two infants in the BB-12 group, could be explained by osmotic diarrhoea when xylitol is ingested. But since the daily xylitol dose in the present study was only $200 \mathrm{mg}$, this is unlikely.

BB-12 has been shown to survive in intestinal passage in a dose-dependent manner ${ }^{(26)}$. However, a relatively high variation of recovery rates from individual to individual was observed. Faecal recovery of DNA of the ingested probiotic strain in the present study was used as a measure for the successful delivery and intestinal passage of the probiotic. Most of the children in the BB-12 group showed a positive recovery result. The negative results can be explained by the fact that the detection limit of the faecal recovery of BB-12 was rather high. The possible reason for the faecal recovery of BB-12 in the control group could be maternal consumption of dairy probiotic products containing undefined mixtures of bifidobacteria or breastfeeding. The assay method that we used is subspecies specific but not strain specific. Maternal breast milk and intestinal bifidobacteria, including species belonging to the $B$. animalis group, guide the compositional development of the Bifidobacterium microbiota in infants ${ }^{(27)}$. BB-12 and other probiotics have shown to reside only transiently in faecal samples ${ }^{(10,28,29)}$
It could be hypothesised that xylitol, the bulk agent of the tablet, could act as a prebiotic substance consequently influencing gut colonization. In theory, xylitol is able to fulfil all classification criteria for a prebiotic food ingredient as defined by Gibson \& Roberfroid ${ }^{(30)}$. In a colon simulator experiment, exposure of the microbial community to a high concentration of xylitol (2\%) revealed prebiotic properties in $x$ litol ${ }^{(31)}$. In the present study, most children received a daily dose of $200 \mathrm{mg}$ xylitol. Only about $10 \%$ of the children received the larger dose, $600 \mathrm{mg}$, after the age of 6 months. Thus, the amounts of xylitol ingested by the infants should be too small by far for xylitol to function as a prebiotic substance.

Pacifiers have been used in the past to deliver medicines but also functional foods such as $x \operatorname{litol}^{(32)}$. Most slowrelease pacifiers have a thick, hard base, and the administration of the active agents takes place through an opening in the front plate ${ }^{(32)}$. Our soft and broad new slow-release pacifier has been developed to contain a pouch in which the food supplement tablet can be inserted. The delivery method was tested before the study began: the BB-12xylitol tablet and the control xylitol tablet dissolved from the pouch of the pacifier both slowly and completely during 7-15 min of sucking ${ }^{(12)}$. Thus, the probiotic tablet could be delivered in a controlled way with the new pacifier. The families participating in the study were interviewed in order to examine the feasibility of the delivery method. Of the families, $85 \%$ reported that the insertion of the probiotic tablet into the pouch of the pacifier was easy. The recommended frequency of administration, twice a day, was regarded as acceptable by $85 \%$ of the families ( $\mathrm{T}$ Taipale, K Pienihäkkinen, $\mathrm{P}$ Alanen et al., unpublished results).

Clinical trials with larger numbers of infants are required to corroborate the aforementioned findings. For logistical purposes, in order to avoid interruption due to study subjects moving to another area, as well as to ensure a uniform socio-economic background, this kind of study is optimally performed in a small community such as Muurame or Korpilahti. The disadvantage is the low number of children born in such areas. We recruited for as long as it was possible, in practice for 2.5 years, and ended up with 109 infants who formed the probiotic group ( $n 55)$ and control group ( $n$ 54). Seventeen children in both the groups did not receive the tablet before the age of 2 months, resulting in thirty-eight children in the probiotic group and thirtyseven children in the control group who started the intervention. However, in spite of the arduousness of the study, very few families left the study. Also the fact that a high percentage of families used the tedious spoon-feeding method to deliver the tablet speaks for the low rate of attrition. In the power calculations, we estimated that we would need approximately forty children to start the intervention. Our infant number was lower than anticipated. The number was, however, high enough to detect a $17 \%$ absolute risk reduction, which is statistically significant. 
This kind of reduction in the disease occurrence is also clinically significant. Studies with similar subject numbers have shown significant differences in the occurrence of $\mathrm{AOM}^{(15)}$. Perhaps due to successful breastfeeding, the children participating in the present study were remarkably healthy, apart from respiratory infections. Thus, for the other infectious and non-infectious diseases, the number of infants should have been higher to be able to detect any differences between the groups. On the other hand, with regard to these diseases, the present study did not detect even a trend for differences between the groups.

To our knowledge, the present study is the first to report probiotic administration by means of a slow-release pacifier. This modality may offer a safe and controlled way to reduce early respiratory infections.

\section{Acknowledgements}

The trial was designed by T. T., E. S., P. A. and J. J. E. I. provided the information needed to prepare the questionnaires and diaries. K. P. was responsible for data analysis. C. L. and E. B. were responsible for the faecal analysis of BB-12. All the authors participated in writing the report. The excellent assistance of the research nurses Mari Saastamoinen and Tuula Salopuro and the laboratory technicians Karin Schlichter and Masoumeh Taremi is gratefully acknowledged. T. T. had no conflicts of interest. He was supported by personal grants from the Emil Aaltonen and Sohlberg Foundations, Finnish Dental Society Apollonia and the Finnish Dental Association. The funding sources had no involvement in study design, interpretation of data, writing the paper or the decision to submit the paper for publication. Chr. Hansen A/S (Hoersholm, Denmark) donated the BB-12 for the probiotic tablets and helped in carrying out the faecal analysis of BB-12. The tablets were manufactured by Oy Karl Fazer Ab (Vantaa, Finland). The pacifiers were manufactured by Mekalasi Oy (Konnevesi, Finland). Neither Hansen, Fazer nor Mekalasi provided financial support for this clinical study.

\section{References}

1. Vesa S, Kleemola M, Blomqvist S, et al. (2001) Epidemiology of documented viral respiratory infections and acute otitis media in a cohort of children followed from two to twenty-four months of age. Pediatr Infect Dis J 20, 574-581.

2. Alho OP, Koivu M, Sorri M, et al. (1991) The occurrence of acute otitis media in infants. A life-table analysis. Int J Pediatr Otorhinolaryngol 21, 7-14.

3. Kilpi T, Kero J, Jokinen J, et al. (2002) Common respiratory infections early in life may reduce the risk of atopic dermatitis. Clin Infect Dis 34, 620-626.

4. Rautava S, Arvilommi H \& Isolauri E (2006) Specific probiotics in enhancing maturation of IgA responses in formula-fed infants. Pediatr Res 60, 221-224.

5. Holt PG \& Jones CA (2000) The development of the immune system during pregnancy and early life. Allergy 55, 688-697.
6. Gilliland SE, Morelli L \& Reid G (2001) Health and Nutritional Properties of Probiotics in Food Including Powder Milk With Live Lactic Acid Bacteria. Rome: WHO. ftp:// ftp.fao.org/es/esn/food/probio_report_en.pdf (accessed August 25 2008).

7. Rautava S (2007) Potential use of probiotics in the neonate. Semin Fetal Neonatal Med 12, 45-53.

8. Hatakka K \& Saxelin M (2008) Probiotics in intestinal and non-intestinal infectious diseases - clinical evidence. Curr Pharm Des 14, 1351-1367.

9. Floch MH, Walker AW, Guandalini S, et al. (2008) Recommendations for probiotic use - 2008. J Clin Gastroenerol 42, Suppl. 2, 104-108.

10. Fukushima Y, Kawata Y, Hara H, et al. (1998) Effect of a probiotic formula on intestinal immunoglobulin A production in healthy children. Int J Food Microbiol 42, 39-44.

11. Szajewska H \& Mrukowicz JZ (2001) Probiotics in the treatment and prevention of acute infectious diarrhea in infants and children: a systematic review of published randomized, double-blind, placebo-controlled trials. J Pediatr Gastroenterol Nutr 33, Suppl. 2, 17-25.

12. Taipale T, Pienihäkkinen K, Alanen P, et al. (2007) Dissolution of xylitol from a food supplement administered with a novel slow-release pacifier: preliminary results. Eur Arch Paediatr Dent 8, 123-125.

13. Saavedra JM, Abi-Hanna A, Moore N, et al. (2004) Long-term consumption of infant formulas containing live probiotic bacteria: tolerance and safety. Am J Clin Nutr 79, 261-267.

14. Hatakka K, Blomgren K, Pohjavuori S, et al. (2007) Treatment of acute otitis media with probiotics in otitis-prone children - a double-blind, placebo-controlled randomised study. Clin Nutr 26, 314-321.

15. Rautava S, Salminen S \& Isolauri E (2009) Specific probiotics in reducing the risk of acute infections in infancy - a randomised, double-blind, placebo-controlled study. Br J Nutr $\mathbf{1 0 1}$ $1722-1726$.

16. Agostoni C, Braegger C, Decsi T, et al. (2009) Breast-feeding: a commentary by the ESPGHAN committee on nutrition. $J$ Pediatr Gastroenterol Nutr 49, 112-125.

17. Hasunen K \& Ryynänen S (2005) Infant feeding in Finland 2005. In Reports of the Ministry of Social Affairs and Health, vol. 19, pp. 1236-2115. Helsinki: Oy Edita Ab.

18. Harmsen HJM, Wildeboer-Veloo ACM, Raangs GC, et al. (2000) Analysis of intestinal flora development in breastfed and formula-fed infants by using molecular identification and detection methods. I Pediatr Gastroenterol Nutr 30, 61-67.

19. Weizman Z, Asli G \& Alsheikh A (2005) Effect of a probiotic infant formula on infections in child care centers: comparison of two probiotic agents. Pediatrics 115, 5-9.

20. Leyer GJ, Li S, Mubasher ME, et al. (2009) Probiotic effects on cold and influenza-like symptom incidence and duration in children. Pediatrics 124, E172-E179.

21. Isolauri E, Kalliomäki M, Laitinen K, et al. (2008) Modulation of the maturing gut barrier and microbiota: a novel target in allergic disease. Curr Pharm Des 14, 1368-1375.

22. North K, Fleming P, Golding J, et al. (1999) Pacifier use and morbidity in the first six months of life. Pediatrics 103, E34.

23. Niemelä M, Pihakari O, Pokka T, et al. (2000) Pacifier as a risk factor for acute otitis media: a randomized, controlled trial of parental counselling. Pediatrics 106, 483-488.

24. Chaves RG, Lamounier JA \& Cesar CC (2007) Factors associated with duration of breastfeeding. J Pediatr (Rio J) $\mathbf{8 3}$, $241-246$

25. Uhari M, Kontiokari T \& Niemelä M (1998) A novel use of xylitol sugar in preventing acute otitis media. Pediatrics 102, 879-884. 
26. Larsen C, Nielsen S, Kaestel P, et al. (2006) Dose-response study of probiotic bacteria Bifidobacterium animalis subsp. lactis BB-12 and Lactobacillus paracasei subsp. paracasei CRL-341 in healthy young adults. Eur I Clin Nutr $\mathbf{6 0}$, 1284-1293.

27. Grönlund MM, Gueimonde M, Laitinen K, et al. (2007) Maternal breast-milk and intestinal bifidobacteria guide the compositional development of the Bifidobacterium microbiota in infants at risk of allergic disease. Clin Exp Allergy 37, 1764-1772.

28. Saxelin M, Pessi T \& Salminen S (1995) Fecal recovery following oral administration of Lactobacillus Strain GG (ATCC 53103) in gelatine capsules to healthy volunteers. Int J Food Microbiol 25, 199-203.
29. Isolauri E, Rautava S, Kalliomäki M, et al. (2002) Role of probiotics in food hypersensitivity. Curr Opin Allergy Clin Immunol 2, 263-271.

30. Gibson GR \& Roberfroid MB (1995) Dietary modulation of the human colonic microbiota: introducing the concept of prebiotics. J Nutr 125, 1401-1412.

31. Mäkeläinen HS, Mäkivuokko HA, Salminen SJ, et al. (2007) The effects of polydextrose and xylitol on microbial community and activity in a 4-stage colon simulator. J Food Sci $\mathbf{7 2}$, M153-M159.

32. Aaltonen AS, Suhonen JT, Tenovuo J, et al. (2000) Efficacy of a slow-release device containing fluoride, xylitol and sorbitol in preventing infant caries. Acta Odontol Scand 58, $285-292$. 